\title{
Bose Mott-Insulators as Closed Shells
}

\author{
A. F. R. de Toledo Piza \\ Departamento de Física Matemática, \\ Instituto de Física, Universidade de São Paulo, \\ C.P. 66318, 05315-970 São Paulo, S.P., Brazil
}

Received on 16 December, 2004

\begin{abstract}
Properties of the ground-state, zero temperature 'phase diagram' of the Bose-Hubbard hamiltonian as understood by Fisher et al. [5] are studied using simple tools in the case of finite systems. The results obtained provide a transparent picture of the thermodynamic limit and reveal features reminiscent of shell structure in many-fermion systems. Independent sites and many-site correlations appear in correspondence with independent fermions and many-fermion correlations respectively. This paper is dedicated to Nicim Zagury, on the occasion of his seventieth birthday.
\end{abstract}

\section{Introduction}

Perhaps the most satisfying justification of the simplicity of dilute (gaseous), single species Bose-Einstein condensates (BEC's) has been provided not more than a couple of years ago by Lieb and Seiringer[1], by proving that the ground state of a finite many-boson system confined to an external 'trap' represented by a confining potential $V_{\text {trap }}(\vec{r})$ is, in the limit when the number of bosons $N$ increases to infinity in such a way that the product $N a$ remains constant, $a$ being the (positive) scattering length associated with a two-boson, purely repulsive interaction potential, is such that its exact one body density matrix is of the form

$$
\rho\left(\vec{r}, \vec{r}^{\prime}\right)=N \phi_{0}(\vec{r}) \phi_{0}^{*}\left(\vec{r}^{\prime}\right) .
$$

The function $\phi_{0}(\vec{r})$ is the minimizer of the Gross-Pitaevski functional

$$
W[\phi] \equiv \int d^{3} r \phi^{*}(\vec{r})\left(\frac{-\hbar^{2} \nabla^{2}}{2 M}+V_{\text {trap }}(\vec{r})+\frac{\lambda N}{2}|\phi(\vec{r})|^{2}\right) \phi(\vec{r}) .
$$

and this limit is accordingly called the Gross-Pitaevski (GP) limit. The quantity $\lambda \equiv 4 \pi \hbar^{2} a / M$ plays the role of the strength of an effective, zero range two-body interaction. Note that it is expressed in terms of the scattering length $a$, and is thus an object possessing the hierarchy of the twobody scattering amplitude, as e.g. in Brueckner theory[2]. The confined many-boson ground state becomes therefore exactly a 'single-mode' state in this G-P limit. Even though the size of the system remains finite as $N$ goes to infinity (since the mean field like contribution involving the product $N \lambda$ remains constant, implying that the particle density $\rho$ also becomes infinite), the system is in fact extremely dilute in the sense that the dimensionless quantity $\rho a^{3}$ goes to zero as $N^{-2}$. Now, at least qualitatively, this limit can be said to be "close" to typical gaseous BECs now routinely produced in many laboratories, even though the precise meaning of being close (i.e., within the framework of ref.[1]) has still to be worked out. These gases are such that they satisfy the hierarchy of length scales

$$
a \ll \rho^{-1 / 3} \ll(\rho a)^{-1 / 2}
$$

i.e., the characteristic distance associated with the interaction much smaller than the mean interparticle spacing, which is in turn much smaller than the so called 'healing length' [3]. These inequalities are in fact equivalent to $\rho a^{3} \ll 1$.

In the real life gaseous BECs one has typically $\rho a^{3} \sim$ $10^{-6}$, so that corrections to the extreme single-mode, no depletion picture for the condensate state are apt to be small. The correction for the energy per particle first derived by Lee and Yang in 1957[4] for a uniform system of particle density $\rho$,

$$
\frac{E}{N}=\frac{1}{2} N \lambda \rho\left(1+\frac{128}{15 \sqrt{\pi}} \sqrt{\rho a^{3}}\right)
$$

and the standard estimate for the depletion of the condensate based on Bogoliubov theory (see e.g. ref.[3]) 


$$
\bar{N}=\frac{8 N}{3 \sqrt{\pi}} \sqrt{\rho a^{3}}
$$

are consistent with the general picture of ref.[1] and may be taken at least as heuristic estimates of the deviations from the extreme single mode picture to be expected in the real gaseous systems. Note that in the G-P limit $\rho a^{3} \rightarrow 0$ as $N^{-2}$. It should also be kept in mind that the actual two-body interaction is not purely repulsive. As a result of this, the minimizer of the Gross-Pitaevski functional in fact approximates a state which is unstable against the so called threebody recombination processes. A relative slowness of the effects of such processes is therefore a crucial requirement in the handling of these Bose-Einstein condensed states.

The single mode picture emerging in the G-P limit applies also to ground states in which the external trap potential consists of a periodic array of local minima separated by quantum-mechanically permeable barriers. In this case, the single mode picture entails full delocalization of the trapped bosons. Note however that the mean occupation of any local minimum diverges in the G-P limit, so that one is led to ask what new physics gets deployed as one keeps short of it (i.e., finite, and possibly even small mean occupations of individual minima), in the case of such traps. In this case an heuristic answer can be found in the context of the Bose-Hubbard (B-H) model, analyzed from the point of view of statistical mechanics before the experimental achievement of dilute atomic Bose-Einstein condensates, notably by Fisher et al.[5].

In fact, the Bose-Hubbard model can be seen as a manymode approximation to the cold dilute system of bosonic atoms involving the states in the lowest band of the periodic potential array[6]. By using site-localized (Wanier) combinations of the lowest band modes as a single-boson basis, and noting that i) matrix elements of the one-body part of the effective hamiltonian are strongly dominated by nearest neighbor matrix elements, and ii) matrix elements of the effective two-body interaction are strongly dominated by single site two-body matrix elements, one is led immediately to the Bose-Hubbard hamiltonian

$$
H_{\mathrm{BH}}=-f \sum_{\langle m, n\rangle}\left(a_{m}^{\dagger} a_{n}+a_{n}^{\dagger} a_{m}\right)+\frac{J}{2} \sum_{n} a_{n}^{\dagger} a_{n}^{\dagger} a_{n} a_{n}
$$

where the creation-annihilation operators correspond to the site localized single-boson states, the second sum runs over all the different sites while the first sum runs over nearest neighbor site pairs. The positive constants $f$ and $J$ stand for the relevant one and two body matrix elements (a repulsive effective two body interaction being assumed).

In what follows the heuristic derivation of the "zero temperature phase diagram" of this model given by Fisher et al.[5] will be implemented in the case of a finite number $M$ of sites and of small numbers of bosons per site. Many relevant properties of such a finite system actually be obtained analytically in a simple way, shedding revealing light on some aspects of the corresponding thermodynamic limit, which consists in letting $M \rightarrow \infty$ with constant number of bosons per site. Some heuristic contact with the different G-P limit will also be provided. The finite B-H system itself will be shown to admit an interpretation in terms of the physics of quantum shell effects, familiar in fermionic systems such as atoms and atomic nuclei. This paper is dedicated to Nicim Zagury, on the occasion of his seventieth birthday.

\section{Finite Bose-Hubbard ground states}

In order to proceed with the analysis of properties of the ground states of the hamiltonian (1), periodic boundary conditions will be used for the one-dimensional array of $M$ sites, so that the nearest neighbors of site $n=M$ are the sites $n=M-1$ and $n=1$. In this case the hopping term of $H_{B H}$ becomes diagonal if one introduces an alternate representation in terms of the "momentum" single boson states defined as

$$
A_{q} \equiv \frac{1}{\sqrt{M}} \sum_{n=1}^{M} e^{\frac{2 \pi i}{M} n q} a_{n}, \quad q=0, \ldots, M-1
$$

The hamiltonian (1) can then be rewritten as

$$
\begin{gathered}
H_{B H}=-2 f \sum_{q=0}^{M-1} \cos \left(\frac{2 \pi q}{M}\right) A_{q}^{\dagger} A_{q} \\
+\sum_{q_{j}} \delta_{M}\left(q_{1}+q_{2}-q_{3}-q_{4}\right) A_{q_{3}}^{\dagger} A_{q_{4}}^{\dagger} A_{q_{2}} A_{q_{1}}
\end{gathered}
$$

where the modular Kronecker delta $\delta_{M}(q)$ is equal to one if $q=0, \pm M, \pm 2 M, \ldots$ and zero otherwise. This shows moreover that, in addition to the total number of bosons $N \equiv \sum_{n} a_{n}^{\dagger} a_{n} \equiv \sum_{q} A_{q}^{\dagger} A_{q}$, the total "momentum" is also a (modular) constant of motion and can be used to label the eigenstates of $H_{B H}$.

\subsection{Independent sites}

Following the analysis given in ref. [5] consider first the case $f=0$. Here the representation in terms of the localized orbitals diagonalizes the hamiltonian, as

$$
H_{B H} \rightarrow \frac{J}{2} \sum_{n} N_{n}\left(N_{n}-1\right), \quad N_{n} \equiv a_{n}^{\dagger} a_{n}
$$

Introducing the zero temperature grand potential $\Omega \equiv$ $H_{B H}-\mu N$, where $\mu$ is the chemical potential, one has

$$
\Omega=\frac{J}{2} \sum_{n}\left(N_{n}-\frac{\mu}{J}-\frac{1}{2}\right)^{2}-\frac{M J}{2}\left(\frac{\mu}{J}+\frac{1}{2}\right)^{2}
$$


so that, for the case of $N=n_{0} M+\nu$ bosons, $0 \leq \nu \leq$ $M-1$, the value of $\Omega$ corresponding to the lowest eigenvalue of $H_{B H}$ is

$$
\begin{aligned}
& \frac{\Omega_{0}(N)}{J}=\frac{M}{2}\left(n_{0}-\frac{\mu}{J}-\frac{1}{2}\right)^{2} \\
& +\nu\left(n_{0}-\frac{\mu}{J}\right)-\frac{M}{2}\left(\frac{\mu}{J}+\frac{1}{2}\right)^{2} .
\end{aligned}
$$

The values of $\Omega_{0}(N) / J$ for given $\mu / J$ and varying $N$ lie along a polygonal line with vertices at the "commensurate filling" values $n_{0} M$ and with the nonzero values of $\nu$ along the straight sides. The number of particles which minimizes $\Omega_{0}(N) / J$ is controlled by the chemical potential $\mu / J$. This number is unique and corresponds to a commensurate filling situation except when $\mu / J$ is an integer $n_{0}$, in which case the two commensurate fillings $n_{0}$ and $n_{0}+1$ together with all the intermediate $\nu$ values correspond to the same minimum value of $\Omega_{0}(N) / J$. This pattern is illustrated in Fig. 1(a). (a)

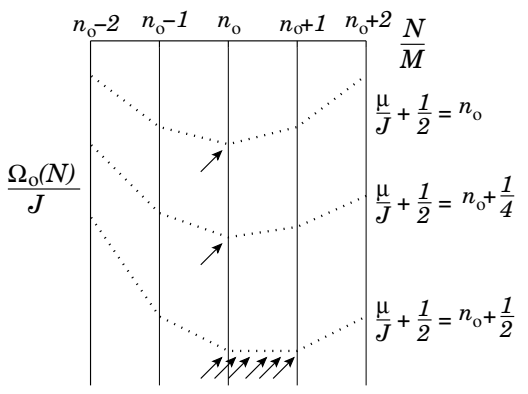

(b)

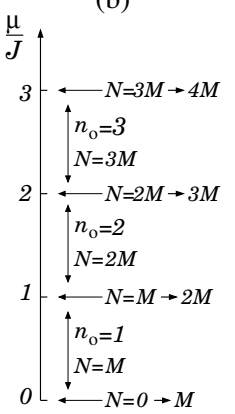

Figure 1. (a)-values of $\Omega_{0}(N) / J$ as a function of the number of particles $N$ for different values of the chemical potential $\mu$. The minima (indicated by slanted arrows) are unique for each value of $\mu$ and correspond to a commensurate filling situations except when $\mu / J$ has an integer value, in which case degeneracy occurs, as shown; (b)-alternate representation giving the number(s) of bosons which minimize(s) $\Omega_{0}(N) / J$ along a $\mu / J$ axis.
The usual way to plot this result as part of a "zero temperature phase diagram" consists in marking along an $\mu / J$ axis the number of particles which minimizes the grand potential. This is shown in Fig. 1(b). The minimizing number $N$ is clearly independent of $\mu / J$ in open intervals between successive integer values. In these intervals one gets therefore the "incompressibility" relation

$$
\frac{\partial N_{\min }}{\partial \mu}=0, \quad N_{\min }=n_{0} M, \quad n_{0}<\frac{\mu}{J}<n_{0}+1 .
$$

Note that an alternate way to describe this situation without explicit reference to the grand potential consists in evaluating the $f=0$ ground state eigenvalues for $N=n_{0} M+\nu$

$$
E_{0}^{(f=0)}(N)=\frac{J}{2} M\left(n_{0}-\frac{1}{2}\right)^{2}+\nu J n_{0}-\frac{J}{8}
$$

and evaluating the one boson addition energy in units of $J$

$$
\frac{E_{0}^{(f=0)}(N+1)-E_{0}^{(f=0)}(N)}{J}=n_{0}
$$

which shows unit jumps at successive commensurate fillings.

\subsection{Perturbative effects of hopping}

The ground states for commensurate fillings $n_{0}$ in the limit $f=0$ are non-degenerate and easily written down in terms of the localized creation operators $a_{n}^{\dagger}$ as

$$
\left|\left\{n_{0}\right\}^{M}\right\rangle=\prod_{n=1}^{M}\left(\frac{a_{n}^{\dagger} n_{0}}{\sqrt{n_{0} !}}\right)|0\rangle .
$$

The lowest order perturbative correction to the energy of these states due to hopping effects is clearly of order $f^{2}$. The $f=0$ ground states for $N=n_{0} M \pm 1$, on the other hand, are $M$-fold degenerate, allowing for non-vanishing first order hopping corrections to their energies. They can be written as

$$
\begin{array}{llrl}
N=n_{0} M+1: & \frac{1}{\sqrt{n_{0}+1}} a_{n}^{\dagger}\left|\left\{n_{0}\right\}^{M}\right\rangle, & n=1, \ldots, M ; \\
N=n_{0} M-1: & \frac{1}{\sqrt{n_{0}}} a_{n}\left|\left\{n_{0}\right\}^{M}\right\rangle, & n=1, \ldots, M .
\end{array}
$$

The diagonalization of the hopping (first) term of the hamiltonian (1) or (3) within these degenerate subspaces can be achieved in a straightforward way in terms of the momentum single boson states associated to the operators (2). In fact, direct calculations give

$$
\begin{aligned}
& -\frac{2 f}{n_{0}+1} \sum_{q}\left\langle\left\{n_{0}\right\}^{M}\left|A_{p_{1}} A_{q}^{\dagger} A_{q} A_{p_{2}}^{\dagger}\right|\left\{n_{0}\right\}^{M}\right\rangle \cos \left(\frac{2 \pi q}{M}\right)=-2 f\left(n_{0}+1\right) \cos \left(\frac{2 \pi p_{1}}{M}\right) \delta_{p_{1} p_{2}} ; \\
& -\frac{2 f}{n_{0}+1} \sum_{q}\left\langle\left\{n_{0}\right\}^{M}\left|A_{p_{1}}^{\dagger} A_{q}^{\dagger} A_{q} A_{p_{2}}\right|\left\{n_{0}\right\}^{M}\right\rangle \cos \left(\frac{2 \pi q}{M}\right)=-2 f n_{0} \cos \left(\frac{2 \pi p_{1}}{M}\right) \delta_{p_{1} p_{2}},
\end{aligned}
$$


so that the appropriate zeroth order eigenstates involve respectively the creation and the annihilation of one boson in a momentum state on the commensurate occupation state. The first order ground state energy shifts correspond to $p_{1}=0$ in each case, leading to the first order corrections to the addition energies

$$
\begin{aligned}
& \frac{E\left(n_{0} M+1\right)-E\left(n_{0} M\right)}{J}=n_{0}-2\left(n_{0}+1\right) \frac{f}{J}+\mathcal{O}\left(f^{2}\right), \\
& \frac{E\left(n_{0} M\right)-E\left(n_{0} M-1\right)}{J}=\left(n_{0}-1\right)+2 n_{0} \frac{f}{J}+\mathcal{O}\left(f^{2}\right) .
\end{aligned}
$$

If one adds to the graph of Fig. 1(b) an additional axis for the scaled hopping parameter $f / J$, the linear terms define respectively minimal and maximal slopes of curves branching out of the integer values of the $\mu / J$ axis. Note that when one more boson is added to a state with commensurate filling $n_{0}$, one obtains the minimal slope branching out of the value of $\mu / J$ which constitutes the upper bound of the incompressibility domain of this commensurate filling state; removal of one boson from the commensurate filling state $n_{0}$, on the other hand, gives the maximal slope branching out of the lower incompressibility limit of this state. This is shown on the left hand side of Fig. 2, where one sees that the maximal and minimal slopes branching out of each integer value $n \geq 1$ on the $\mu / J$ axis are in fact equal except for sign, and given by $\pm 2(n+1)$. These results are independent of $M$, and therefore survive in the thermodynamic limit $M \rightarrow \infty$ with fixed $n_{0}$.

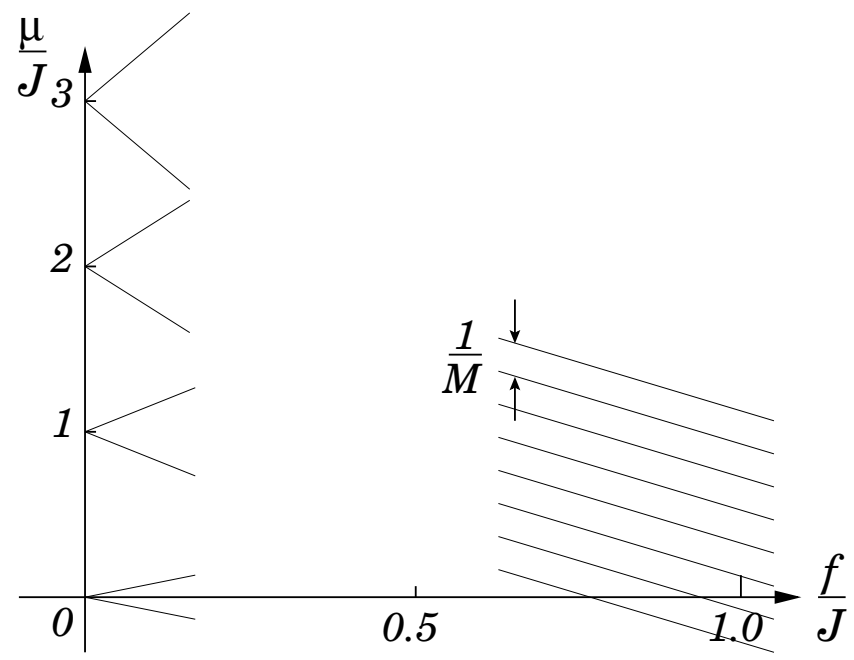

Figure 2. On the left hand side the calculated limiting slopes of $\mu / J$ as a function of $f / J$ at very small hopping are shown. On the right hand side the calculated slopes and spacings of $\mu / J$ curves for large values of $f / J$ are shown for several consecutive values of the total number of bosons $N$. The number of sites is $M$.

\subsection{The limit of very strong hopping}

The situation prevailing at the far right along the $f / J$ axis can also be easily worked out in closed form. Here the on site effective two-body interaction can be handled perturbatively, the unperturbed, non-degenerate $N$ boson ground state being given as the "pure hopping" Perron-Frobenius ground state

$$
\left|N_{\text {hop }}\right\rangle=\frac{1}{\sqrt{N !}}\left(A_{0}^{\dagger}\right)^{N}|0\rangle,
$$

for which the energy is, to first order in $J$ (see eq. (3)),

$$
E(N)=-2 f N+\frac{J}{2 M} N(N-1) .
$$

The properly scaled addition energies are therefore

$$
\frac{E(N+1)-E(N)}{J}=\frac{1}{M} N-2 \frac{f}{J},
$$

showing that all the staggering found in the small hopping limit has disappeared, leading to a family of equally spaced lines with slope -2 , their spacing being $M$-dependent and given by $1 / M$. They become therefore more dense as one approaches the thermodynamic limit, where the spacing eventually vanishes. Updating Fig. 2 with this additional information leads to the picture shown, which now gives a fairly clear image of the complete diagram.

While this cannot be obtained analytically, the smoothness of the missing intermediate filling can be checked within computer workable limits of $M$ and $N$ by means of straight numerical diagonalization of the Bose-Hubbard hamiltonian $H_{B H}$. Note that the dimensionality of the matrices that have to be handled can be significantly reduced by taking into account the constants of motion of this hamiltonian. An example of the result of such numerical approach is shown in Fig. 3. Salient features are the progressive shortening of the "incompressible lobes" in the $f / J$ direction as $n_{0}$ becomes larger, which is already indicated by the values of the initial extremal slopes calculated in the $f \rightarrow 0$ limit, and the closing of these lobes in the thermodynamic limit where the uniform width $1 / M$ of the various parallel channels eventually shrinks to zero. 


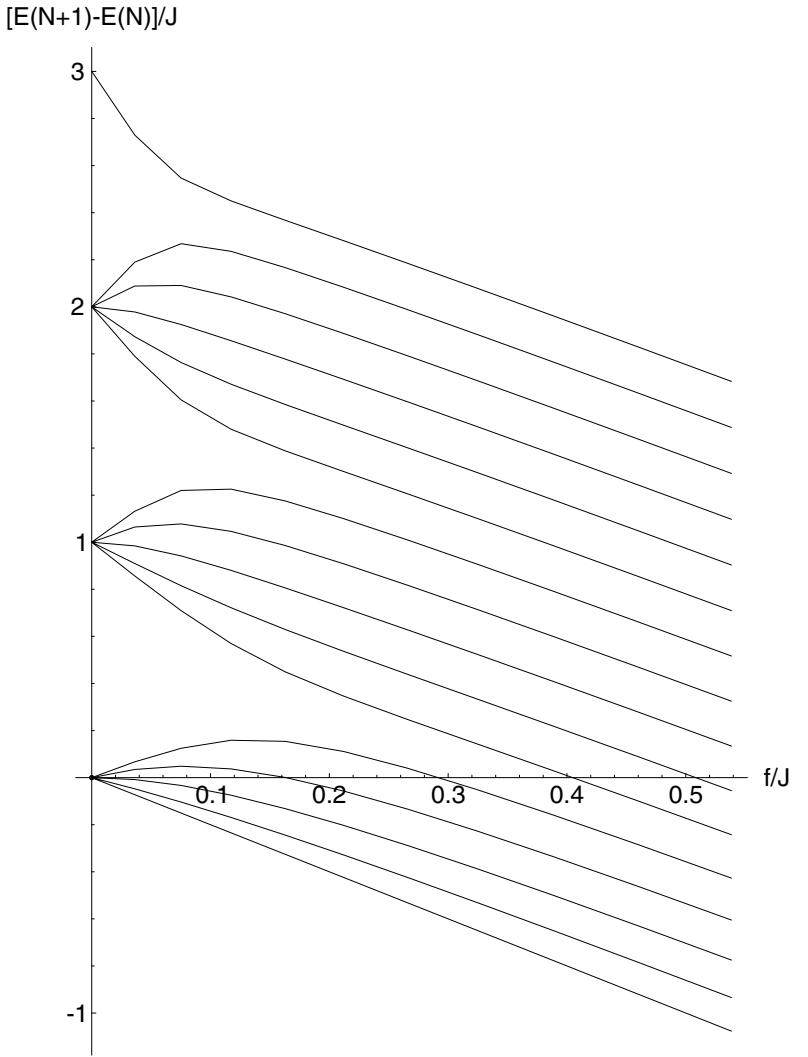

Figure 3. Numerical zero temperature "phase diagram" for the Bose-Hubbard hamiltonian with $M=5$ and periodical boundary conditions. The chemical potential is replaced by addition energies in units of the on-site two-body interaction parameter $J$. The first three Mott-insulator lobes are shown.

It may be noted at this point that the Gross-Pitaevski limit of Lieb and Seiringer leads to an entirely different situation when compared with the thermodynamic limit as used above. It corresponds in fact to letting $J \rightarrow 0$ and $N \rightarrow \infty$ in such a way as to preserve the Gross-Pitaevski functional, implying therefore constant, non-vanishing $f$ when the different sites are limited by barriers of finite height. The Gross-Pitaevski domain therefore lies at the extreme upper right region of the zero temperature phase diagram, where the delocalization of the bosons is always complete. In this region the ground state of the Bose-Hubbard model is essentially a pure condensate in the delocalized state (2) of zero "momentum" as written in eq. (5), in qualitative agreement with the result of Lieb and Seiringer.

\section{One-body features of correlated eigenstates}

Except in the extreme limits $f \rightarrow 0(J \neq 0)$ and $J \rightarrow 0$ $(f \neq 0)$ the eigenstates of the Bose-Hubbard hamiltonian for finite number of sites $m$ and of bosons $N$ contain non obvious many boson correlations due to the combined effects of hopping and of on-site two body interactions. When periodic boundary conditions are used, however, the available conserved quantities allow for a very simple general characterization of the effects of such correlations for onebody observables, in terms of properties of the reduced onebody densities associated with the energy eigenstates. In fact, from the conservation of total momentum (see eq. (3)) it follows that the energy eigenvectors can be chosen to be also eigenvectors of the total momentum, $\left|E_{n}, P_{\text {tot }}\right\rangle$, with $P_{\text {tot }}=0, \ldots, M-1$. Consequently, the reduced one-body density is diagonal in the momentum representation, i.e.

$$
\rho_{q_{1} q_{2}}^{\left(E_{n}, P_{\text {tot }}\right)}=\left\langle E_{n}, P_{\text {tot }}\left|A_{q_{2}}^{\dagger} A_{q_{1}}\right| E_{n}, P_{\text {tot }}\right\rangle=\nu_{q_{1}} \delta_{q_{1} q_{2}} .
$$

In fact, the operators $A_{q_{2}}^{\dagger} A_{q_{1}}$ with $q_{2} \neq q_{1}$ change the value of the total momentum and therefore have vanishing expectation value in states of good total momentum. The $\nu_{q}$ are occupation numbers for the delocalized, momentum single boson states associated with the operators (2), which are therefore the natural orbitals of the reduced one body density. The occupation numbers satisfy the usual trace relation for the one body density

$$
\sum_{q=0}^{M-1} \nu_{q}=N .
$$

A first, trivial example of this is the one body density associated with the commensurate ground state in the $f=0$ limit, eq. (4), for which one easily obtains

$$
\left\langle\left\{n_{0}\right\}^{M}\left|A_{q_{2}}^{\dagger} A_{q_{1}}\right|\left\{n_{0}\right\}^{M}\right\rangle=n_{0} \delta q_{1} q_{2},
$$

showing that the reduced one body density is in fact a multiple of the unit matrix in this case (and therefore diagonal in any representation). Slightly less trivial cases are the normalized, commensurate filling states plus an added particle (or "hole") with momentum $q$,

$$
\left|\left\{n_{0}\right\}^{M}, q\right\rangle \equiv \frac{1}{\sqrt{n_{0}+1}} A_{q}^{\dagger}\left|\left\{n_{0}\right\}^{M}\right\rangle
$$

and

$$
\left|\left\{n_{0}\right\}^{M}, \bar{q}\right\rangle \equiv \frac{1}{\sqrt{n_{0}}} A_{q}\left|\left\{n_{0}\right\}^{M}\right\rangle .
$$

The small $f$, perturbative ground states considered in section 2.2 are states of this type with $q=0$. The corresponding one-body densities can also be evaluated analytically in a straightforward manner with the results 


$$
\begin{aligned}
\left\langle\left\{n_{0}\right\}^{M}, q\left|A_{q_{2}}^{\dagger} A_{q_{1}}\right|\left\{n_{0}\right\}^{M}, q\right\rangle & =\left[n_{0}-\frac{n_{0}}{M}+\left(n_{0}+1\right) \delta_{q_{1} q}\right] \delta_{q_{1} q_{2}}, \\
\left\langle\left\{n_{0}\right\}^{M}, \bar{q}\left|A_{q_{2}}^{\dagger} A_{q_{1}}\right|\left\{n_{0}\right\}^{M}, \bar{q}\right\rangle & =\left[n_{0}-\frac{n_{0}+1}{M}+n_{0} \delta_{q_{2} q}\right] \delta_{q_{1} q_{2}} .
\end{aligned}
$$
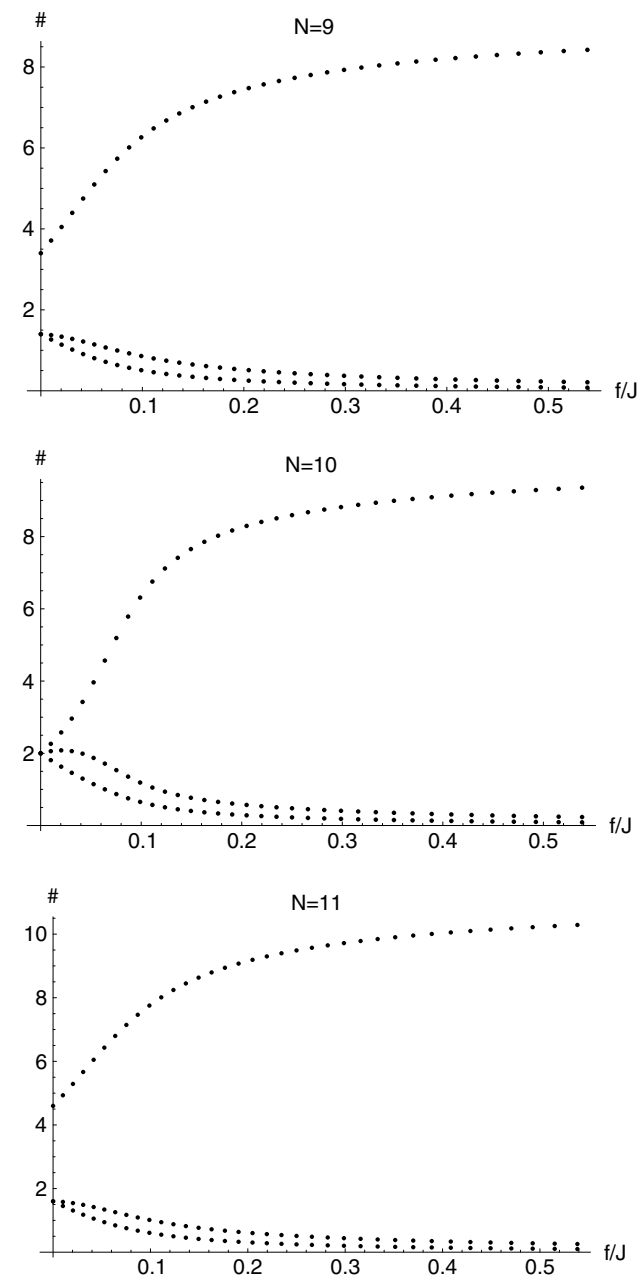

Figure 4. Natural orbital occupation numbers \# plotted against $f / J$ for the ground states of the Bose-Hubbard model with $M=5$ and the indicated total number of bosons $N$. Thus $N=10$ corresponds to commensurate filling with $n_{0}=2$. In each case, the upper branch corresponds to the zero "momentum" natural orbital, while the two lower branches are doubly degenerate corresponding to natural orbitals with "momenta" $q=1,4$ and 2, 3 respectively, and must be counted twice to obtain the total number of bosons.

In both cases one gets for the one body density expressions which consist of the sum of a multiple of the identity matrix plus an additional pure density associated with the momentum state of the additional (or missing) particle. This additional component is enhanced by the corresponding bosonic factors (respectively $n_{0}+1$ and $n_{0}$ ), while the multiple of identity is correspondingly depleted, in order to make up for the required trace property.

The interest of these simple results consists in that they reveal the nature of the coherence properties of the states which can be probed by one body observables. From the fact that the momentum states are the natural orbitals it follows, in fact, that they will be related in general to the associated, delocalized single particle states. For states such as the commensurate filling states the coherence properties are in fact rendered invisible by occupation degeneracy. On the other hand, the stationary states, at very small hopping, resulting from the addition (or removal) of a single particle from these states, will sustain coherence properties of the momentum single particle states enhanced by bosonic factors, superimposed to an incoherent background associated with the "residual" component with degenerate occupancy.

It is a simple matter to evaluate reduced one-body densities numerically for stationary states involving a small enough number of sites and/or particles. Some sample results for the ground states of a system with number of sites $M=5$ and with $N=9,10$ and 11 respectively are given in Fig. 4 for sequences of values of the relative strength of the hopping parameter, $f / J$. In the extreme $f / J=0$ the commensurate filling case with $N=10$ shows a fully degenerate occupancy, while the cases $n=9$ and $N=11$ clearly show the bosonic enhancement in the occupation number of the zero "momentum" natural orbital. Increasing the relative hopping parameter leads in all cases to strong dominance of zero momentum occupancy in the one-body density leading to near saturation for values of $f / J$ in the range corresponding to the "closing" of the corresponding incompressibility lobe in the ground state phase diagram (see Fig. 3).

\section{Alternate picture of the zero tem- perature diagram related to "shell effects"}

An amusing alternate way of displaying the above results consists in plotting the addition energies in units of $J$, $[E(N+1)-E(N)] / J$ against the number of bosons per site $N / M$ for various values of the relative hopping parameter $f / J$. In the extreme case when $f / J=0$ one gets the staircase diagram shown as Fig. 5(a). This behavior is replaced by a smooth linear ramp in the other extreme case when $f / J$ becomes very large. In intermediate cases the steps become progressively 'worn' as the value of $f / J$ is increased and also for the larger values of $N / M$. The last two cases are illustrated in Fig. 5(c) and (b) respectively. This is of course reminiscent of the standard pattern which is associated with fermionic systems displaying shell effects[7]. Fig. 5(a) corresponds to a very strong shell effect which is progressively obliterated as $f / J$ and $N / M$ increase. 


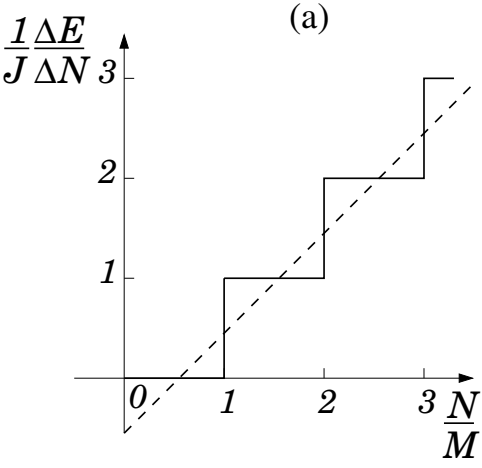

(d)

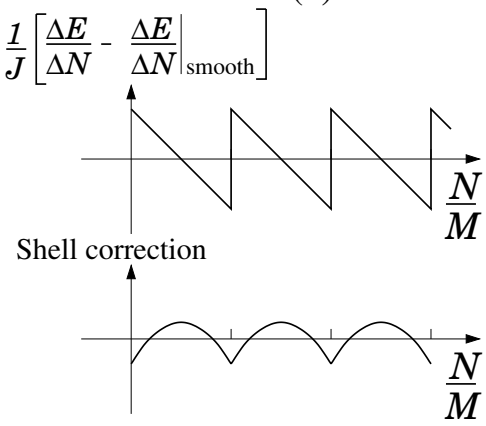

(b)

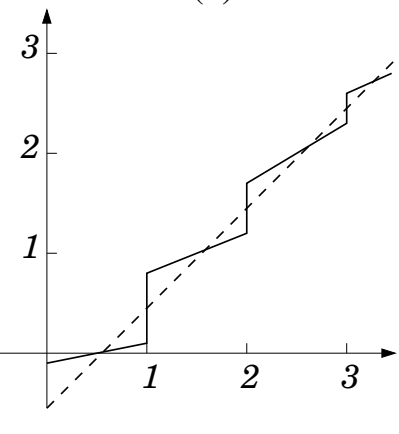

(e)
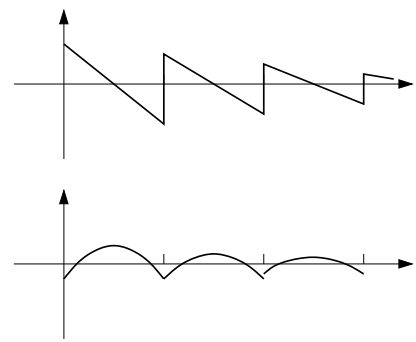

(c)

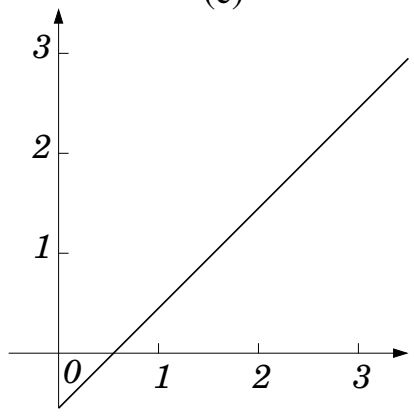

Figure 5. Plots of addition energies $\Delta E / \Delta N=E(N+1)-E(N)$ in units of $J$ against the number of bosons per site $N / M$ for $f / J=0$ (a), $f / J$ small (b) and $f / J$ large (c). The top graphs in (d) and (e) show the "shell effect" corresponding to (a) and (b) respectively, obtained by subtracting the smoothened addition energies. The lower graphs in (d) and (e) show the qualitative behavior of the shell correction to the total energy, obtained by integrating the shell effect over $N / M$.

The "shell effect" can be extracted by plotting the difference between the staggered addition energies and their smoothened average displaying simple ramp behavior. The "shell correction", reflecting deviations of the total energy from the value obtained from the averaged addition energies is then obtained by integrating the shell effect with respect to $N / M$. An integration constant is chosen so that the shell correction has zero average. The behavior of these quantities is illustrated in Fig. 5(d) and (e). The values of $N / M$ for which the shell correction is most negative correspond to "magic numbers", which here correspond to commensurate filling situations (integer $N / M$ ), and hence to the incompressible Mott insulator phase.

We are thus led to a picture in which the incompressible lobes of the zero temperature phase diagram of Fisher correspond to the magic numbers of a many-boson system displaying shell effects to the extent that they occupy many independent (or nearly independent) sites. This, in particular, provides the degeneracies (or near degeneracies) which generate the magic numbers. Increasing the relative hopping parameter reduces the independence of the sites by promoting many-site correlations eventually obliterating the shell structure. In contrast, shell effects in many fermion systems signal independent (or nearly independent) particles, the magic numbers resulting from single particle level degeneracies (or near degeneracies). Increasing many-particle correlations tends to obliterate the fermionic shell effects.

\section{Concluding remarks}

The Bose-Hubbard model for finite number of sites and of bosons is soluble, in the usual sense that exact solutions can be obtained by diagonalizing finite matrices. Numerical results obtained by such means can be rendered quite transparent by using elementary analytical perturbative techniques in the extreme domains in which one has either $f \ll J$ or, conversely, $J \ll f$, since the 'unperturbed' problem admits simple analytical solutions in each of these two cases.

The results one obtains analyzing in this way the zero temperature phase diagram, discussed by Fisher et al. [5] in the thermodynamic limit in which both the number of sites $M$ and the number of bosons $N$ increase to infinity at constant 'density' $N / M$, provide for a "blown up" version of the behaviors prevailing in each of the various parameter domains. From these results one can in particular form suggestive pictures of the closing of the incompressibility lobes corresponding in the thermodynamic limit to the Mottinsulator phase and also of the penetration down to the zero hopping limit of a "superconducting" phase, in the case of incommensurate occupations. A suggestive ingredient in the latter case is the bosonic enhancement effect of added or removed bosons from the commensurate occupation situation.

Finally, an interpretation in terms of "shell effects" suggests itself in terms of which the commensurate filling 
cases for not too large values of $f / J$ correspond to "closed shells". This shell structure is related to nearly independent sites and is obliterated by increasing the relative hopping parameter, so that the corresponding magic numbers are dictated by the number of sites. This is in correspondence with shell stricture in fermionic systems, related to the near validity of an independent particle picture which is obliterated by correlations induced by interparticle interactions, the magic numbers being dictated by energy bunching of single-fermion states.

\section{References}

[1] E. H. Lieb and R. Seiringer, Phys. Rev. Lett. 88, 170409 (2002).
[2] K. A. Brueckner and K. Sawada, Phys. Rev. 106, 1117 and 1128 (1957); K. Sawada, Phys. Rev. 116, 1344 (1959).

[3] A. J. Leggett, Revs. Mod. Phys. 73, 307 (2001).

[4] T. D. Lee and C. N. Yang, Phys. Rev. 105, 1119 (1957).

[5] Matthew P. A. Fisher, Peter B. Weichman, G. Grinstein, and Daniel S. Fisher, Phys. Rev. B 40, 546 (1989).

[6] D. Jaksch, C. Bruder, J. I. Cirac, C. W. Gardiner, and P. Zoller, Phys. Rev. Lett. 81, 3108 (1998).

[7] W. D. Myers and W. J. Swiatecki, Nucl. Phys. 81, 1 (1966); Ark. Fys. 36, 343 (1967). 